Communications in Applied Geometry.

ISSN 2249-4286 Volume 6, Number 1 (2019) pp. 1-7

(C) Research India Publications

https://dx.doi.org/10.37622/CAG/6.1.2019.1-7

\title{
On Classes of Generalized Ruscheweyh Type Hormonic Functions
}

\author{
Rashmi B. $\mathbf{T}^{\mathbf{1}}$ and Dileep $\mathrm{L}^{\mathbf{2}}$ \\ ${ }^{1}$ Assistant Professor, Department of Mathematics, \\ BGS Institute of Technology, Adichunchanagiri university, BG Nagara, Mandya \\ 571448, India. \\ ${ }^{2}$ Associate Professor, Department of Mathematics, \\ Vidyavardhaka College of Engineering, Mysuru-570 002, India.
}

\begin{abstract}
In this paper, we define and investigate the classes of complex valued harmonic functions using the generalized Rucsheweyh derivative $\mathrm{D}_{\lambda}^{\mathrm{n}}$. For functions belonging to these classes, we determine certain necessary and sufficient coefficient conditions. Furthermore, distributions bounds, extreme points, inclusion relations, convolution conditions and convex combinations are also obtained.
\end{abstract}

2000 Mathematics Subject Classification: 30C45.

Key words and phrases: Univalent functions, analytic functions, Jacobian, harmonic functions and generalized Ruscheweyh derivative

\section{INTRODUCTION}

A complex-valued continuous function $\omega=f(z)=u(z)+i v(z)$ defined on a domain $\mathcal{G}$ is harmonic if both $u$ and $v$ are real-valued harmonic functions on $\mathcal{G}$, that is $u$ and $v$ satisfy, respectively the Laplace equations

$$
\Delta u=u_{x x}+u_{y y}=0 \text { and } \Delta v=v_{x x}+v_{y y}=0 \text {. }
$$

A one to one mapping $u=u(z), v=v(z)$ from a region $\mathcal{G}_{1}$ in the xy-plane to a region $\mathcal{G}_{2}$ in the uv-plane is a harmonic mapping if $u$ and $v$ are harmonic. It is well known that if $f=u+i v$ has continuous partial derivatives, then $f$ is analytic if and 
only if the Cauchy-Riemann equations $u_{x}=v_{y}$ and $u_{y}=-v_{x}$ are satisfied. It follows that every analytic function is a complex-valued harmonic function. However , not every complex-valued harmonic functions is analytic, since no two solutions of the Laplace equation can be taken as the components $u$ and $v$ of an analytic function in $\mathcal{G}$, they must be related by the Cauchy-Riemann equations $u_{x}=v_{y}$ and $u_{y}=-v_{x}$.

A subject of considerable importance in harmonic mappings is the Jacobian.

If

$$
J_{f}(z)=\left|f_{z}(z)\right|^{2}-\left|f_{\bar{z}}(z)\right|^{2}=\left|h^{\prime}(z)\right|^{2}-\left|g^{\prime}(z)\right|^{2}
$$

wheref $=h+\bar{g}$ is harmonic function in $\mathcal{U}=\{z:|z|<1\}$. When $J_{f}$ is positive in $\mathcal{U}$, the harmonic function $f$ is called orientation -preserving or sense-preserving in $\mathcal{U}$. A harmonic mapping is locally univalent in a neighborhood of a point $z_{0}$ if and only if $J_{f}(z) \neq 0$ at $z_{0}$.

The first key insight into harmonic univalent mappings came from Clunie and Small [2] who observed that $f=h+\bar{g}$ is locally univalent and orientation pre-serving if and only if

$$
J_{f}(z)=\left|h^{\prime}(z)\right|^{2}-\left|g^{\prime}(z)\right|^{2}>0 \text {. }
$$

This is equivalent to

$$
\left|g^{\prime}(z)\right|<\mid h^{\prime}(z)
$$

Let $\mathcal{H}$ denote the family of all harmonic, complex-valued orientation preserving, normalized and univalent mapping defined on $\mathcal{U}$. Thus, a function $f$ in $\mathcal{H}$ admits the representation $f=h+\bar{g}$, where,

$$
h(z)=z+\sum_{j=2}^{\infty} a_{j} z^{j} \text { and } g(z)=\sum_{j=1}^{\infty} b_{j} z^{j},\left|b_{1}\right|<1 .
$$

Note that the family $\mathcal{H}$ of orientation preserving normalized, harmonic univalent functions reduces to the class $S$ of normalized analytic univalent functions if the co-analytic part of $f=h+\bar{g}$ is analytically zero. Further, let $\overline{\mathcal{H}}$ denote the subfamily of $\mathcal{H}$ consists of harmonic functions $f=h+\bar{g}$ so that $h$ and $g$ are of the form

$$
h(z)=z+\sum_{j=2}^{\infty}\left|a_{j}\right| z^{j} \text { and } g(z)=\sum_{j=1}^{\infty}\left|b_{j}\right| z^{j},\left|b_{1}\right|<1 .
$$

The generalized Ruschweyh derivative $D_{\lambda}^{n} f$ [1] is defined as follows:

For $f \in \mathcal{A}(n), \lambda \geq 0$ and $n \in \mathbb{R}, n>-1$, we have

$$
D_{\lambda}^{n} f(z)=\frac{z}{(1-z)^{n+1}} * D_{\lambda} f(z), \quad z \in \mathcal{U},
$$


where, $D_{\lambda} f(z)=(1-\lambda) f(z)+\lambda z f^{\prime}(z)$.

For $=h+\bar{g}$, given by (1.1), we define the generalized Ruscheweyh derivative of the harmonic function by,

$$
D_{\lambda}^{n} f(z)=D_{\lambda}^{n} h(z)+\overline{D_{\lambda}^{n} g(z)}
$$

The power series expansion of $D_{\lambda}^{n} f(z)$ is of the form

$$
D_{\lambda}^{n} f(z)=z+\sum_{j=2}^{\infty} C_{\lambda}(n, j) a_{j} z^{j}
$$

where,$C_{\lambda}(n, j)=[1+(j-1) \lambda] \frac{(n+1)_{j-1}}{(1)_{j-1}}$.

Using the generalized Ruscheweyh derivative, we define the subclass $\mathcal{F}_{H}(n, \alpha, \beta, \lambda)$ as the family of harmonic functions $f=h+\bar{g}$ of the form (1.1), such that

$$
\Re\left\{(1-\alpha)\left[\frac{D_{\lambda}^{n} f(z)}{z}\right]+\alpha\left[\frac{\frac{\partial}{\partial \theta} D_{\lambda}^{n} f(z)}{\frac{\partial z}{\partial \theta}}\right]\right\}>\beta, \alpha \geq 0,
$$

where, $0 \leq \beta<1$ and $z=r e^{i \theta} \in \mathcal{U}$.

Further, $\mathcal{F}_{\bar{H}}(n, \alpha, \beta, \lambda)=\mathcal{F}_{H}(n, \alpha, \beta, \lambda) \cap \overline{\mathcal{H}}$. As $\alpha$ changes from 0 to 1 , the family $\mathcal{F}_{H}(n, \alpha, \beta, \lambda)$ produces a passage from the class of harmonic functions $\mathcal{P}_{H}(n, \beta, \lambda) \equiv$ $\mathcal{F}_{H}(n, 0, \beta, \lambda)$ consisting of harmonic functions $f$ where $\Re\left\{\left[\frac{D_{\lambda}^{n} f(z)}{z}\right]\right\}>\beta$ to the class of harmonic functions

$\mathcal{Q}_{H}(n, \beta, \lambda) \equiv \mathcal{F}_{H}(n, 1, \beta, \lambda)$ consisting of harmonic functions $f$ where

$$
\left\{\frac{\frac{\partial}{\partial \theta} D_{\lambda}^{n} f(z)}{\frac{\partial z}{\partial \theta}}\right\}>\beta .
$$

\section{PRIME RESULTS}

In this section, certain sufficient condition for $f=h+\bar{g}$ given by (1.1) to be in the class $\mathcal{F}_{H}(n, \alpha, \beta, \lambda)$ and $\mathcal{F}_{\bar{H}}(n, \alpha, \beta, \lambda)$ are obtained. Further, a representation theorem, inclusion properties and distortion bounds are also obtained for functions belonging to class $\mathcal{F}_{\bar{H}}(n, \alpha, \beta, \lambda)$.

Theorem 2.1: Let $f=h+\bar{g}$ be given by (1.1). If (2.1) $\sum_{j=2}^{\infty} C_{\lambda}(n, j)|\alpha(j-1)+1|\left|a_{j}\right|+\sum_{j=1}^{\infty} C_{\lambda}(n, j)|\alpha(j+1)-1|\left|b_{j}\right| \leq(1-\beta)$ then $f \in \mathcal{F}_{H}(n, \alpha, \beta, \lambda)$. 
Proof. Let

$\omega(z)=(1-\alpha)\left[\frac{D_{\lambda}^{n} f(z)}{z}\right]+\alpha\left\{\frac{\frac{\partial}{\partial \theta} D_{\lambda}^{n} f(z)}{\frac{\partial z}{\partial \theta}}\right\}$

It suffices to show that $|1-\beta+\omega| \geq|1+\beta-\omega|$. This is equivalent to show that if the condition (2.1), holds then,

$$
\begin{gathered}
(1-\beta) z+(1-\alpha)\left[D_{\lambda}^{n} h(z)+\overline{D_{\lambda}^{n} g(z)}\right]+\alpha\left[z\left(D_{\lambda}^{n} h(z)\right)^{\prime}-z\left(\overline{D_{\lambda}^{n} g(z)}\right)^{\prime}\right] \\
-\left|(1+\beta) z-(1-\alpha)\left[D_{\lambda}^{n} h(z)+\overline{D_{\lambda}^{n} g(z)}\right]-\alpha\left[z\left(D_{\lambda}^{n} h(z)\right)^{\prime}-z\left(\overline{D_{\lambda}^{n} g(z)}\right)^{\prime}\right]\right|: M(\alpha, \beta) \geq 0 .
\end{gathered}
$$

Substituting for $D_{\lambda}^{n} h(z)$ and $D_{\lambda}^{n} g(z)$ in $M(\alpha, \beta)$ yields.

$$
\begin{aligned}
M(\alpha, \beta) & =\left|(1-\beta) z+\left\{\sum_{j=2}^{\infty}(\alpha(j-1)+1) a_{j} z^{j}-\sum_{j=1}^{\infty}(\alpha(j+1)-1) \bar{b}_{j}(\bar{z})^{j}\right\} C_{\lambda}(n, j)\right| \\
& \left.-\mid \beta z-\left\{\sum_{j=2}^{\infty}(\alpha(j-1)+1) a_{j} z^{j}-\sum_{j=1}^{\infty}(\alpha(j+1)-1)-\bar{b}_{j}(\bar{z})^{j}\right\} C_{\lambda}(n, j)\right\} \mid \\
& \geq 2|z|\left[(1-\beta)-\left(\sum_{j=2}^{\infty}|\alpha(j-1)+1|\left|a_{j}\right|+\sum_{j=1}^{\infty}|\alpha(j+1)-1|\left|b_{j}\right|\right) C_{\lambda}(n, j)\right] .
\end{aligned}
$$

By (2.1), it follows that the last expression is non-negative. Hence $f \in \mathcal{F}_{H}(n, \alpha, \beta, \lambda)$.

We derive a necessary and sufficient condition for function $f=h+\bar{g}$ be given with (1.4).

Theorem 2.2. Let $f=h+\bar{g}$, be given by (1.1). then, $f \in \mathcal{V}_{\bar{H}}(n, \alpha, \lambda)$ if and only if

$$
\sum_{j=2}^{\infty}\left(\frac{j-\alpha}{1-\alpha}\left|a_{j}\right|+\frac{j+\alpha}{1-\alpha}\left|b_{j}\right|\right) C_{\lambda}(n, j) \leq 1-\frac{1+\alpha}{1-\alpha} b_{1}
$$

where,$a_{1}=1$ and $0 \leq \alpha<1$.

Proof: $\operatorname{Since} \mathcal{V}_{\bar{H}}(n, \alpha, \lambda) \subset \mathcal{R}_{H}(n, \alpha, \lambda)$, we only need to prove the necessity part of the Theorem. For functions $f \in \mathcal{V}_{\bar{H}}(n, \alpha, \lambda)$, we notice that the condition

$$
\frac{\partial}{\partial \theta}\left(\arg D_{\lambda}^{n} f(z)\right) \geq \alpha
$$


is equivalent to

$$
\frac{\partial}{\partial \theta}\left(\arg D_{\lambda}^{n} f(z)\right)-\alpha=\mathcal{R}\left\{\frac{z\left(D_{\lambda}^{n} h(z)\right)^{\prime}-\overline{z\left(D_{\lambda}^{n} g(z)\right)^{\prime}}}{z\left(D_{\lambda}^{n} h(z)\right)-z\left(D_{\lambda}^{n} g(z)\right)}-\alpha\right\} \geq 0 .
$$

That is,

$$
\mathcal{R}\left[\frac{(1-\alpha) z+\left(\sum_{j=2}^{\infty} C_{\lambda}(n, j)(j-\alpha)\left|a_{j}\right| z^{j}-\sum_{j=1}^{\infty} C_{\lambda}(n, j)(j+\alpha)\left|b_{j}\right| \overline{z^{J}}\right)}{z+\left(\sum_{j=2}^{\infty} C_{\lambda}(n, j)\left|a_{j}\right| z^{j}+\sum_{j=1}^{\infty} C_{\lambda}(n, j)\left|b_{j}\right| \overline{z^{j}}\right)}\right] \geq 0 .
$$

The above condition must hold for all values of $z \in \mathcal{U}$. Choosing $\phi$ according to (1.4), we must have,

$$
\text { (2.4) } \frac{(1-\alpha)-(1+\alpha) b_{1}-\left(\sum_{j=2}^{\infty}(j-\alpha) C_{\lambda}(n, j)\left|a_{j}\right| r^{j-1}+\sum_{j=2}^{\infty}(j+\alpha) C_{\lambda}(n, j)\left|b_{j}\right| r^{j-1}\right)}{1+\left|b_{1}\right|+\left(\sum_{j=2}^{\infty} C_{\lambda}(n, j)\left|a_{j}\right|+\sum_{j=2}^{\infty} C_{\lambda}(n, j)\left|b_{j}\right|\right) r^{j-1}} \geq 0 \text {. }
$$

If the condition (2.2) does not hold, then the numerator in (2.4) is negative for $r$ sufficiently close to 1 . Hence there exists $z_{0}=r_{0} \in(0,1)$ for which quotient of (2.4) is negative. This contradiction proves that $f \in \mathcal{V}_{\bar{H}}(n, \alpha, \lambda)$.

Theorem 2.3: If $f \in \mathcal{V}_{\bar{H}}(n, \alpha, \lambda)$, then

$$
(2.5)|f(z)| \leq\left(1+\left|b_{1}\right|\right) r+C_{\lambda}(n, 2)\left(\frac{1-\alpha}{2-\alpha}-\frac{1+\alpha}{2-\alpha}\left|b_{1}\right|\right) r^{2}, \quad(|z|=r<1)
$$

and

$$
|f(z)| \geq\left(1+\left|b_{1}\right|\right) r-C_{\lambda}(n, 2)\left(\frac{1-\alpha}{2-\alpha}-\frac{1+\alpha}{2+\alpha}\left|b_{1}\right|\right) r^{2}, \quad(|z|=r<1) .
$$

Proof: We will prove the inequality (2.6). The argument for (2.5) is similar. Let $f \in \mathcal{V}_{\bar{H}}(n, \alpha, \lambda)$. Taking the absolute values of $f$, we have

$$
\begin{gathered}
|f(z)| \leq\left(1+\left|b_{1}\right|\right) r+\sum_{j=2}^{\infty}\left(\left|a_{j}\right|+\left|b_{j}\right|\right)|r|^{j} \\
\leq\left(1+\left|b_{1}\right|\right) r+r^{2} \sum_{j=2}^{\infty}\left(\left|a_{j}\right|+\left|b_{j}\right|\right) .
\end{gathered}
$$


That is

$$
\begin{aligned}
&|\mathrm{f}(\mathrm{z})| \leq(1+\left.\left|\mathrm{b}_{1}\right|\right) \mathrm{r} \\
&+\frac{1-\alpha}{(2-\alpha) \mathrm{C}_{\lambda}(\mathrm{n}, 2)}\left(\sum_{j=2}^{\infty} \frac{(2-\alpha)}{(1-\alpha)} C_{\lambda}(n, 2)\left|a_{j}\right|\right. \\
&\left.+\sum_{j=2}^{\infty} \frac{(2-\alpha)}{(1-\alpha)} C_{\lambda}(n, 2)\left|b_{j}\right|\right) r^{2} \\
& \leq\left(1+\left|b_{1}\right|\right) r+\frac{1-\alpha}{(2-\alpha) C_{\lambda}(n, 2)}\left[1-\frac{(1+\alpha)}{(1-\alpha)}\left|b_{1}\right|\right] r^{2} \\
& \leq\left(1+\left|\mathrm{b}_{1}\right|\right) r+\frac{1}{\mathrm{C}_{\lambda}(\mathrm{n}, 2)}\left(\frac{1-\alpha}{2-\alpha}-\frac{1+\alpha}{2-\alpha}\left|b_{1}\right|\right) r^{2} .
\end{aligned}
$$

Corollary 2.4: Let $f$ be the form (1.1), so that $f \in \mathcal{V}_{\bar{H}}(n, \alpha, \lambda)$.Then,

(2.7) $\left\{\omega:|\omega|<\frac{2 C_{\lambda}(n, 2)-1-\left[C_{\lambda}(n, 2)-1\right] \alpha}{(2-\alpha) C_{\lambda}(n, 2)}-\frac{2 C_{\lambda}(n, 2)-1-\left[C_{\lambda}(n, 2)-1\right] \alpha}{(2+\alpha) C_{\lambda}(n, 2)} b_{1}\right\} \subset f(\mathcal{U})$.

We use the coefficient bounds to examine the extreme points for $f \in \mathcal{V}_{\bar{H}}(n, \alpha, \lambda)$ and determine extreme point of $\mathcal{V}_{\bar{H}}(n, \alpha, \lambda)$.

Theorem 2.5: The extreme points for $\mathcal{V}_{\bar{H}}(n, \alpha, \lambda)$ are

$$
\left\{z+\gamma_{j} x z^{j}+\overline{b_{1} z}\right\} \cup\left\{z+\overline{b_{1} z+\mu_{\jmath} x z^{\jmath}}\right\}
$$

where, $j \geq 2$ and $|x|=1-\left|b_{1}\right|$

$$
\gamma_{j}=\frac{(1-\alpha)}{(j-\alpha) C_{\lambda}(n, j)} \text { and } \mu_{j}=\frac{(1+\alpha)}{(j+\alpha) C_{\lambda}(n, j)} .
$$

Proof. Any functions $f$ in $\mathcal{V}_{\bar{H}}(n, \alpha, \lambda)$ is of the form

$$
f(z)=z+\sum_{j=2}^{\infty}\left|a_{j}\right| e^{i \beta_{j}} Z^{j}+\overline{b_{1} z}+\overline{\sum_{J=2}^{\infty}\left|b_{J}\right| e^{i \delta_{J}} Z^{J}}
$$


where the coefficients satisfy the inequality (2.1).

Let $h_{1}(z)=z, \quad g_{1}(z)=b_{1}(z), \quad h_{j}(z)=z+\gamma_{j} e^{i \beta_{j} z^{j}}, \quad g_{j}(z)=b_{1} z+\mu_{j} e^{i \delta_{j}} Z^{j}$, for $j=2,3, \ldots$

Writing $\chi_{j}=\frac{\left|a_{j}\right|}{\gamma_{j}} \eta_{j}=\frac{\left|b_{j}\right|}{\mu_{j}} \quad j=2,3, \ldots$

and $\chi_{1}=1-\sum_{j=2}^{\infty} \chi_{j} \eta_{1}=1-\sum_{j=2}^{\infty} \eta_{j}$

we have,

$$
f(z)=\sum_{j=2}^{\infty}\left(\chi_{j} h_{j}(z)+\eta_{j} g_{j}(z)\right) .
$$

In particular, setting

$f_{1}(z)=z+\overline{b_{1} z}$ and $f_{j}(z)=z+\gamma_{j} x z^{j}+\overline{b_{1} z}+\overline{\mu_{\jmath} y z^{\jmath}}, \quad(j \geq 2,|x|+|y|=1-$ $\left.\left|b_{1}\right|\right)$.

We see that the extreme points of $\mathcal{V}_{\bar{H}}(n, \alpha, \lambda)$ are contained in $\left\{f_{j}(z)\right\}$.

\section{REFERENCES}

[1] K. Al-Shaqsi and M.Darus, On certain subclass of analytic univalent functions with negative coefficients, Appl. Math.Sci., Vol. 1 (2007), No.3, 1121-1128.

[2] J. Clunie and T. Sheil-Small, Harmonic univalent functions, Ann. Acad. Aci. Fenn. Ser. A. I.Math. , 9( 1984), 3-25.

[3] J. M. Jahangiri and H. Silverman, Harmonic univalent functions with varyig arguments, Int.J. Appl. Math., 8, No.3, (2002), 267-275.

[4] G. Murugusundaramoorthy, A class of Ruscheweyh type harmonic univalent functions, South-west Journal of Pure and Applied Mathematics, Issue 2, (2003), 90-95.

[5] H. Silverman, Univalent functions with varying arguments, Houston J. Math., 7(1981), 283-289. 
